\title{
The Category of Partial Doi-Hopf Modules and Functors
}

\author{
Q.-G. Chen (*) - D.-G. WANG $(* *)$
}

Abstract - Let $(H, A, C),\left(H^{\prime}, A^{\prime}, C^{\prime}\right)$ be two partial Doi-Hopf datums consisting of a Hopf algebra $H$, a partial right $H$-comodule algebra $A$ and a partial right $H$ module coalgebra. Given $\alpha: H \rightarrow H^{\prime}, \beta: A \rightarrow A^{\prime}$ and $\gamma: C \rightarrow C^{\prime}$, we define an induction functor between the category $\mathcal{M}(H)_{A}^{C}$ of all partial Doi-Hopf modules and the category $\mathcal{M}\left(H^{\prime}\right)_{A^{\prime}}^{C^{\prime}}$, and we prove that this functor has a right adjoint. Specially, we then give necessary and sufficient conditions for the functor $F: \mathcal{M}(H)_{A}^{C} \rightarrow \mathcal{M}(H)_{A}$ (exactly the category of right $A$-modules). This leads to a generalized notion of integrals. Moreover, from these results, we deduce a version of Maschke-type Theorems for partial Doi-Hopf modules. The applications of our results are considered.

Mathematics Subject Classification (2010). 16W30.

KEYworDs. Hopf algebras, partial Doi-Hopf modules, integral.

\section{Introduction}

Partial group actions were considered first by Exel [E1] in the context of operator algebras and they turned out to be a powerful tool in the study of $C^{*}$-algebras generated by partial isometries on a Hilbert space [E2]. A treatment from a purely algebraic point of view was given recently in

(*) Indirizzo dell'A.: School of Mathematics and Statistics, Yili Normal College Yining 835000, China.

E-mail: cqg211@163.com

(**) Indirizzo dell'A.: School of Mathematical Sciences, Qufu Normal University, Qufu, Shandong 273165, P.R.China (corresponding author).

E-mail: dingguo95@126.com

The first author was supported by the National Natural Science Foundation of China (No. 11261063) and the Fund of the Key Disciplines in the General Colleges and Universities of Xin Jiang Uygur Autonomous Region (No. 2012ZDXK03). The second anthor was supported by the National Natural Science Foundation of China (No. 11171183) and the Shandong Provincial Natural Science Foundation of China (No. ZR2011AM013). 
[DEP], [DFP], [DZ] and [DE]. Partial Hopf actions were motivated by an attempt to generalize the notion of partial Galois extensions of commutative rings in [DFP] to a broader context. The definition of partial Hopf actions and co-actions were introduced by using the notions of partial entwining structures in [CJ].

The category $\mathcal{M}(H)_{A}^{C}$ of Doi-Hopf modules was introduced in [D], where $H$ is a Hopf algebra, $A$ a right $H$-comodule algebra and $C$ a right $H$ module coalgebra. It is the category of the modules over the algebra $A$ which are also comodules over the coalgebra $C$ and satisfy certain compatibility condition involving $H$. The study of $\mathcal{M}(H)_{A}^{C}$ turned out to be very useful: it was shown in [D] that many categories such as the module and comodule categories over bialgebras, the Hopf modules category [S], Takeuchi's relative Hopf modules, graded modules, modules graded by $G$-sets, Long dimodules and the Yetter-Drinfeld category ([CMI], [RT], [Y]) are special cases of $\mathcal{M}(H)_{A}^{C}$.

As a general version of the category $\mathcal{M}(H)_{A}^{C}$ of Doi-Hopf modules, we shall consider a partial Doi-Hopf datum $(H, A, C)$, and the category $\mathcal{M}(H)_{A}^{C}$ of socalled partial Doi-Hopf modules. The starting point of this paper is an attempt to discuss the results of [CR] in the partial case. This would have meant in particular giving generalizations of the induced and the coinduced functors. In the paper, we give a generalization of the induction functor and try to characterize the separability of the functor $F: \mathcal{M}(H)_{A}^{C} \rightarrow \mathcal{M}(H)_{A}$ which leads to the generalized integral of the partial Doi-Hopf datum $(H, A, C)$. Also, a version of Maschke-type Theorems for partial Doi-Hopf modules is proved.

The paper is organized as follows.

In Section 2, we recall definitions and basic results related to Hopf partial action, and introduce the induction functor: given maps $\alpha: H \rightarrow H^{\prime}, \beta: A \rightarrow A^{\prime}$ and $\gamma: C \rightarrow C^{\prime}$, we have a functor (called induction functor) $F=\bullet \otimes_{A} A^{\prime}: \mathcal{M}(H)_{A}^{C} \rightarrow \mathcal{M}\left(H^{\prime}\right)_{A^{\prime}}^{C^{\prime}}$. This functor have a right adjoint $G$. In Section 3, we discuss the separability of the functor $F: \mathcal{M}(H)_{A}^{C} \rightarrow \mathcal{M}(H)_{A}$ that forgets the $C$-coaction,which leads to a generalized integral of a partial Doi-Hopf datum $(H, A, C)$. The applications of our results are considered in Section 4.

\section{Partial Module Coalgebras, Partial Comodule Algebras, Partial Doi-Hopf Modules}

Throughtout this paper, $k$ will be a field. Unless specified otherwise, all modules, algebras, coalgebras, bialgebras(or Hopf algebras), tensor pro- 
ducts and homomorphisms are over $k . l$ denotes the identity mapping. $H$ will be a Hopf algebra with an invertible antipode $S$ and we will use Sweedler's sigma-notation extensively. For example, if $\left(C, \Delta_{C}, \varepsilon_{H}\right)$ is a coalgebra, then for all $c \in C$, we write

$$
\Delta_{C}(c)=c_{1} \otimes c_{2} \in C \otimes C .
$$

Definition 2.1. Let $H$ be a Hopf algebra. A $k$-algebra $A$ is called a partial right $H$-comodule algebra, if there exists a $k$-linear map $\rho_{A}: A \rightarrow A \otimes H, \rho_{A}(a)=a_{[0]} \otimes a_{[1]}$ such that the following conditions satisfy:

$$
\begin{gathered}
\rho_{A}(a b)=\rho_{A}(a) \rho_{A}(b), \\
\rho_{A}\left(a_{[0]}\right) \otimes a_{[1]}=a_{[0]} 1_{A[0]} \otimes a_{[1] 1} 1_{A[1]} \otimes a_{[1] 2}, \\
\varepsilon\left(a_{[1]}\right)\left(a_{[0]}\right)=a,
\end{gathered}
$$

for all $a, b \in A$.

EXAMPLE 2.2. Let $e \in H$ be an idempotent such that $e \otimes e=$ $\Delta(e)\left(e \otimes 1_{H}\right)$ and $\varepsilon(e)=1$. Then we can define the following partial right $H$-coaction on $A=k: \rho(x)=x \otimes e \in k \otimes H$.

Definition 2.3. Let $H$ be a Hopf algebra. A $k$-coalgebra $C$ is called a partial right $H$-module coalgebra, if there exists a $k$-linear map $\phi: C \otimes H \rightarrow C, \phi(c \otimes h)=c \cdot h$ such that the following conditions satisfy:

$$
\begin{gathered}
(c \cdot h) \cdot g=c \cdot h g, \\
(c \cdot h)_{1} \cdot 1_{H} \otimes(c \cdot h)_{2}=c_{1} \cdot h_{1} \otimes c_{2} \cdot h_{2}, \\
\varepsilon(c \cdot h)=\varepsilon(c) \varepsilon(h),
\end{gathered}
$$

for all $c \in C$ and $g, h \in H$.

ExAmple 2.4. Let $e \in H$ be a central idempotent such that $e \otimes e=$ $\Delta(e)\left(e \otimes 1_{H}\right)$ and $\varepsilon(e)=1$. Then we can define the partial right $H$-action on $C=H: g \cdot h=e g h$.

A partial Doi-Hopf datum is a threetuple $(H, A, C)$, where $H$ is a Hopf algebra, $A$ a partial right $H$-comodule algebra and $C$ a partial right $H$-module coalgebra. Given a partial Doi-Hopf datum $(H, A, C)$. A partial 
Doi-Hopf module $M$ is a right $A$-module and there exists a $k$-linear map $\rho: M \rightarrow M \otimes C$ such that

$$
\rho_{M}^{2}(m)=m_{[0]} \cdot 1_{A[0][0]} \otimes m_{[1] 1} \cdot 1_{A[0][1]} \otimes m_{[1] 2} \cdot 1_{A[1]},
$$

where $\rho_{M}^{2}=\left(\rho_{M} \circ \imath\right) \circ \rho_{M}$,

$$
\begin{gathered}
\rho(m \cdot a)=m_{[0]} \cdot a_{[0]} \otimes m_{[1]} \cdot a_{[1]}, \\
\varepsilon\left(m_{[1]}\right) m_{[0]}=m,
\end{gathered}
$$

for all $m \in M$ and $a \in A$.

ExAmple 2.5. Let $e \in H$ be a central idempotent such that $e \otimes e=$ $\Delta(e)\left(e \otimes 1_{H}\right)$ and $\varepsilon(e)=1$. Then $(H, k, H)$ is a partial Doi-Hopf datum.

$\mathcal{M}(H)_{A}^{C}$ will be the category of partial Doi-Hopf modules and $A$-linear, $C$-colinear maps. Now we can give the following result for the category of partial Doi-Hopf modules which is analogous of [Theorem 1.1, CR].

TheOREM 2.6. Consider two partial Doi-Hopf datums $(H, A, C)$ and $\left(H^{\prime}, A^{\prime}, C^{\prime}\right)$, and suppose that we have maps $\alpha: H \rightarrow H^{\prime}, \beta: A \rightarrow A^{\prime}$ and $\gamma: C \rightarrow C^{\prime}$ which are respectively Hopf algebra, algebra and coalgebra maps satisfying

$$
\begin{gathered}
\gamma(c \cdot h)=\gamma(c) \cdot \alpha(h), \\
\rho_{A}(\beta(a))=\beta\left(a_{[0]}\right) \otimes \alpha\left(a_{[1]}\right),
\end{gathered}
$$

for all $c \in C, h \in H$ and $a \in A$. Then we have a functor $F: \mathcal{M}(H)_{A}^{C} \rightarrow$ $\mathcal{M}\left(H^{\prime}\right)_{A^{\prime}}^{C^{\prime}}$, defined as follows:

$$
F(M)=M \otimes_{A} A^{\prime},
$$

where $A^{\prime}$ is a left $A$-module via $\beta$ and with structure maps defined by

$$
\begin{gathered}
\left(m \otimes_{A} a^{\prime}\right) \cdot b^{\prime}=m \otimes_{A} a^{\prime} b^{\prime}, \\
\rho_{F(M)}\left(m \otimes_{A} a^{\prime}\right)=m_{[0]} \otimes_{A} a_{[0]}^{\prime} \otimes \gamma\left(m_{[1]}\right) \cdot a_{[1]}^{\prime},
\end{gathered}
$$

for all $a^{\prime}, b^{\prime} \in A^{\prime}$ and $m \in M$.

Proof. Let us show that $M \otimes_{A} A^{\prime}$ is an object of $\mathcal{M}\left(H^{\prime}\right)_{A^{\prime}}^{C^{\prime}}$. For this, we need to show that $M \otimes_{A} A^{\prime}$ satisfies conditions (2.7)-(2.9). Notice that 
$M \otimes_{A} A^{\prime}$ satisfies (2.9) obviously. We restrict here to check that $M \otimes_{A} A^{\prime}$ satisfies conditions (2.7) and (2.8). Take $m \in M$ and $a^{\prime}, b^{\prime} \in A^{\prime}$. Then

$$
\begin{aligned}
\rho_{F(M)}\left(\left(m \otimes_{A} a^{\prime}\right) \cdot b^{\prime}\right) & =m_{[0]} \otimes_{A} a_{[0]}^{\prime} b_{[0]}^{\prime} \otimes \gamma\left(m_{[1]}\right) \cdot a_{[1]}^{\prime} b_{[1]}^{\prime} \\
& =\left(m_{[0]} \otimes_{A} a_{[0]}^{\prime}\right) \cdot b_{[0]}^{\prime} \otimes\left(\gamma\left(m_{[1]}\right) \cdot a_{[1]}^{\prime}\right) \cdot b_{[1]}^{\prime},
\end{aligned}
$$

i.e., (2.8) holds. For (2.7), for all $m \in M$ and $a^{\prime} \in A^{\prime}$, we have

$$
\begin{aligned}
& \rho_{F(M)}^{2}\left(m \otimes_{A} a^{\prime}\right) \\
& =m_{[0][0]} \otimes_{A} a_{[0][0]}^{\prime} \otimes \gamma\left(m_{[0][1]}\right) \cdot a_{[0][1]}^{\prime} \otimes \gamma\left(m_{[1]}\right) \cdot a_{[1]}^{\prime} \\
& \stackrel{(2.7)}{=} m_{[0]} \cdot 1_{A[0]} \otimes_{A} a_{[0][0]}^{\prime} \otimes \gamma\left(m_{[1] 1} \cdot 1_{A[1]}\right) \cdot a_{[0][1]}^{\prime} \otimes \gamma\left(m_{[1] 2}\right) \cdot a_{[1]}^{\prime} \\
& =m_{[0]} \otimes_{A} \beta\left(1_{A[0]}\right) a_{[0][0]}^{\prime} \otimes \gamma\left(m_{[1] 1}\right) \cdot \alpha\left(1_{A[1]}\right) a_{[0][1]}^{\prime} \otimes \gamma\left(m_{[1] 2}\right) \cdot a_{[1]}^{\prime} \\
& \stackrel{(2.11)}{=} m_{[0]} \otimes_{A} 1_{A^{\prime}[0]} a_{[0][0]}^{\prime} \otimes \gamma\left(m_{[1] 1}\right) \cdot 1_{A^{\prime}[1]} a_{[0][1]}^{\prime} \otimes \gamma\left(m_{[1] 2}\right) \cdot a_{[1]}^{\prime} \\
& \stackrel{(2.1)}{=} m_{[0]} \otimes_{A} a_{[0][0]}^{\prime} \otimes \gamma\left(m_{[1] 1}\right) \cdot a_{[0][1]}^{\prime} \otimes \gamma\left(m_{[1] 2}\right) \cdot a_{[1]}^{\prime} \\
& =m_{[0]} \otimes_{A} a_{[0]}^{\prime} 1_{A^{\prime}[0][0]} \otimes \gamma\left(m_{[1] 1}\right) \cdot a_{[1] 1}^{\prime} 1_{A^{\prime}[0][1]} \otimes \gamma\left(m_{[1] 2}\right) \cdot a_{[1] 2}^{\prime} 1_{A^{\prime}[1]} \\
& \stackrel{(2.5)}{=}\left(m_{[0]} \otimes_{A} a_{[0]}^{\prime}\right) \cdot 1_{A^{\prime}[0][0]} \otimes\left(\gamma\left(m_{[1]}\right) \cdot a_{[1]}^{\prime}\right)_{1} \cdot 1_{A^{\prime}[0][1]} \otimes\left(\gamma\left(m_{[1]}\right) \cdot a_{[1]}^{\prime}\right)_{2} \cdot 1_{A^{\prime}[1]} .
\end{aligned}
$$

This is exactly what we have to show.

TheOREm 2.7. Under the assumptions of Theorem 2.6, we have a functor $G: \mathcal{M}\left(H^{\prime}\right)_{A^{\prime}}^{C^{\prime}} \rightarrow \mathcal{M}(H)_{A}^{C}$ which is right adjoint to $F$. $G$ is defined by

$$
G\left(M^{\prime}\right)=\overline{M^{\prime} \square_{C^{\prime}} C}=\left\{m^{\prime} \cdot \beta\left(1_{A[0]}\right) \otimes c \cdot 1_{A[1]}\right\},
$$

where $m^{\prime} \otimes c \in M^{\prime} \otimes C$ satisfies the following condition:

$$
\begin{aligned}
m_{[0]}^{\prime} \cdot \beta\left(1_{A[0][0]}\right) \otimes m_{[1]}^{\prime} \cdot \alpha\left(1_{A[0][1]}\right) \otimes c \cdot 1_{A[1]} \\
=m^{\prime} \cdot \beta\left(1_{A[0][0]}\right) \otimes \gamma\left(c_{1}\right) \cdot \alpha\left(1_{A[0][1]}\right) \otimes c_{2} \cdot 1_{A[1]},
\end{aligned}
$$

for all $M^{\prime} \in \mathcal{M}\left(H^{\prime}\right)_{A^{\prime}}^{C^{\prime}}$, and with structure maps

$$
\begin{gathered}
\rho_{G\left(M^{\prime}\right)}\left(m^{\prime} \cdot \beta\left(1_{A[0]}\right) \otimes c \cdot 1_{A[1]}\right)=m^{\prime} \cdot \beta\left(1_{A[0][0]}\right) \otimes c_{1} \cdot 1_{A[0][1]} \otimes c_{2} \cdot 1_{A[1]}, \\
\left(m^{\prime} \cdot \beta\left(1_{A[0]}\right) \otimes c \cdot 1_{A[1]}\right) \cdot a=m^{\prime} \cdot \beta\left(a_{[0]}\right) \otimes c \cdot a_{[1]},
\end{gathered}
$$

for all $a \in A$.

Proof. Let us first show that $G\left(M^{\prime}\right)$ is an object of $\mathcal{M}(H)_{A}^{C}$. It is routine to check that $G\left(M^{\prime}\right)$ is a right $C$-comodule. In order to prove that $M$ is a right $A$-module, we need to show that $m^{\prime} \cdot \beta\left(a_{[0]}\right) \otimes c \cdot a_{[1]} \in \overline{M^{\prime} \square_{C^{\prime}} C}$, for all 
$a \in A$. Indeed,

$$
\begin{aligned}
\left(m^{\prime} \cdot \beta\right. & \left.\left(a_{[0]}\right)\right)_{[0]} \cdot \beta\left(1_{A[0][0]}\right) \otimes\left(m^{\prime} \cdot \beta\left(a_{[0]}\right)\right)_{[1]} \cdot \alpha\left(1_{A[0][1]}\right) \otimes c \cdot a_{[1]} 1_{A[1]} \\
& \stackrel{(2.8)}{=} m_{[0]}^{\prime} \cdot \beta\left(a_{[0]}\right)_{[0]} \beta\left(1_{A[0][0]}\right) \otimes m_{[1]}^{\prime} \cdot \beta\left(a_{[0]}\right)_{[1]} \alpha\left(1_{A[0][1]}\right) \otimes c \cdot a_{[1]} 1_{A[1]} \\
& \stackrel{(2.11)}{=} m_{[0]}^{\prime} \cdot \beta\left(a_{[0][0]}\right) \beta\left(1_{A[0][0]}\right) \otimes m_{[1]}^{\prime} \cdot \alpha\left(a_{[0][1]}\right) \alpha\left(1_{A[0][1]}\right) \otimes c \cdot a_{[1]} 1_{A[1]} \\
& \stackrel{(2.1)}{=} m_{[0]}^{\prime} \cdot \beta\left(1_{A[0][0]}\right) \beta\left(a_{[0][0]}\right) \otimes m_{[1]}^{\prime} \cdot \alpha\left(1_{A[0][1]}\right) \alpha\left(a_{[0][1]}\right) \otimes c \cdot 1_{A[1]} a_{[1]} \\
& \stackrel{(2.14)}{=} m^{\prime} \cdot \beta\left(1_{A[0][0]}\right) \beta\left(a_{[0][0]}\right) \otimes \gamma\left(c_{1}\right) \cdot \alpha\left(1_{A[0][1]}\right) \alpha\left(a_{[0][1]}\right) \otimes c_{2} \cdot 1_{A[1]} a_{[1]} \\
& \stackrel{(2.1)}{=} m^{\prime} \cdot \beta\left(a_{[0][0]}\right) \beta\left(1_{A[0][0]}\right) \otimes \gamma\left(c_{1}\right) \cdot \alpha\left(a_{[0][1]}\right) \alpha\left(1_{A[0][1]}\right) \otimes c_{2} \cdot a_{[1]} 1_{A[1]} \\
& \stackrel{(2.2,2.1)}{=} m^{\prime} \cdot \beta\left(a_{[0]}\right) \beta\left(1_{A[0][0]}\right) \otimes \gamma\left(c_{1}\right) \cdot \alpha\left(a_{[1] 1}\right) \alpha\left(1_{A[0][1]}\right) \otimes c_{2} \cdot a_{[1] 2} 1_{A[1]} \\
& \stackrel{(2.10)}{=} m^{\prime} \cdot \beta\left(a_{[0]}\right) \beta\left(1_{A[0][0]}\right) \otimes \gamma\left(c_{1} \cdot a_{[1] 1}\right) \cdot \alpha\left(1_{A[0][1]}\right) \otimes c_{2} \cdot a_{[1] 2} 1_{A[1]} \\
& \stackrel{(2.5)}{=} m^{\prime} \cdot \beta\left(a_{[0]}\right) \beta\left(1_{A[0][0]}\right) \otimes \gamma\left(\left(c \cdot a_{[1]}\right)_{1}\right) \cdot \alpha\left(1_{A[0][1]}\right) \otimes\left(c \cdot a_{[1]}\right)_{2} \cdot 1_{A[1]} .
\end{aligned}
$$

This is exactly what we have to show.

$G\left(M^{\prime}\right) \in \mathcal{M}(H)_{A}^{C}$ and the functorial properties are checked in a straightforward way. Let us finally show that $G$ is a right adjoint to $F$. Take $M \in \mathcal{M}(H)_{A}^{C}$, we define $\eta_{M}: M \rightarrow G F(M)=\left(\overline{\left.M \otimes_{A} A^{\prime}\right) \square_{C^{\prime}} C}\right.$ as follows: for all $m \in M$,

$$
\eta_{M}(m)=m_{[0]} \otimes_{A} \beta\left(1_{A[0]}\right) \otimes m_{[1]} \cdot 1_{A[1]} .
$$

For all $a \in A$, we have

$$
\begin{aligned}
\eta_{M}(m \cdot a) & =(m \cdot a)_{[0]} \otimes_{A} \beta\left(1_{A[0]}\right) \otimes(m \cdot a)_{[1]} \cdot 1_{A[1]} \\
& \stackrel{(2.8)}{=} m_{[0]} \cdot a_{[0]} \otimes_{A} \beta\left(1_{A[0]}\right) \otimes m_{[1]} \cdot a_{[1]} 1_{A[1]} \\
& =m_{[0]} \otimes_{A} \beta\left(a_{[0]}\right) \beta\left(1_{A[0]}\right) \otimes m_{[1]} \cdot a_{[1]} 1_{A[1]} \\
& \stackrel{(2.1)}{=} m_{[0]} \otimes_{A} \beta\left(1_{A[0]}\right) \beta\left(a_{[0]}\right) \otimes m_{[1]} \cdot 1_{A[1]} a_{[1]} \\
& =\left(m_{[0]} \otimes_{A} \beta\left(1_{A[0]}\right) \otimes m_{[1]} \cdot 1_{A[1]}\right) \cdot a
\end{aligned}
$$

and

$$
\begin{aligned}
\left(\eta_{M} \otimes \imath\right) \circ \rho_{M}(m) & =m_{[0][0]} \otimes_{A} \beta\left(1_{A[0]}\right) \otimes m_{[0][1]} \cdot 1_{A[1]} \otimes m_{[1]} \\
& \stackrel{(2.7)}{=} m_{[0]} \cdot 1_{A[0]}^{\prime} \otimes_{A} \beta\left(1_{A[0]}\right) \otimes m_{[1] 1} \cdot 1_{A[1]}^{\prime} 1_{A[1]} \otimes m_{[1] 2} \\
& =m_{[0]} \otimes_{A} \beta\left(1_{A[0]}^{\prime} 1_{A[0]}\right) \otimes m_{[1] 1} \cdot 1_{A[1]}^{\prime} 1_{A[1]} \otimes m_{[1] 2} \\
& \stackrel{(2.1)}{=} m_{[0]} \otimes_{A} \beta\left(1_{A[0]}\right) \otimes m_{[1] 1} \cdot 1_{A[1]} \otimes m_{[1] 2} \\
& =\rho_{G F(M)} \circ \eta_{M}(m) .
\end{aligned}
$$

So $\eta_{M} \in \mathcal{M}(H)_{A}^{C}$. 
Take $M^{\prime} \in \mathcal{M}\left(H^{\prime}\right)_{A^{\prime}}^{C^{\prime}}$. Then we define $\delta_{M^{\prime}}: F G\left(M^{\prime}\right) \rightarrow M^{\prime}$, where

$$
\delta_{M^{\prime}}\left(\left(m^{\prime} \cdot \beta\left(1_{A[0]}\right) \otimes c \cdot 1_{A[1]}\right) \otimes_{A} a^{\prime}\right)=\varepsilon_{C}(c) m^{\prime} \cdot a^{\prime} .
$$

Notice that $\delta_{M^{\prime}}$ is $A^{\prime}$-linear. That $\delta_{N}$ is $C^{\prime}$-colinear is proved as follows:

$$
\begin{aligned}
& \left.\left(\delta_{M^{\prime}} \otimes \imath\right) \circ\left(\rho_{F G\left(M^{\prime}\right)}\right)\left(m^{\prime} \cdot \beta\left(1_{A[0]}\right) \otimes c \cdot 1_{A[1]}\right) \otimes_{A} a^{\prime}\right) \\
& \quad=\delta_{M^{\prime}}\left(\left(m^{\prime} \cdot \beta\left(1_{A[0][0]}\right) \otimes c_{1} \cdot 1_{A[0] 1]}\right) \otimes_{A} a_{[0]}^{\prime}\right) \otimes \gamma\left(c_{2} \cdot 1_{A[1]}\right) \cdot a_{[1]}^{\prime} \\
& \quad=m^{\prime} \cdot \beta\left(1_{A[0]}\right) a_{[0]}^{\prime} \otimes \gamma\left(c \cdot 1_{A[1]}\right) \cdot a_{[1]}^{\prime} .
\end{aligned}
$$

Applying $\imath \otimes \imath \otimes \varepsilon_{C}$ to (2.14), this yields

$$
\begin{aligned}
m^{\prime} \cdot \beta\left(1_{A[0]}\right) \otimes \gamma\left(c \cdot 1_{A[1]}\right) & =\varepsilon(c) m_{[0]}^{\prime} \cdot \beta\left(1_{A[0]}\right) \otimes m_{[1]}^{\prime} \cdot \alpha\left(1_{A[1]}\right) \\
& =\varepsilon(c) m_{[0]}^{\prime} \cdot \beta\left(1_{A[0]}\right) \otimes m_{[1]}^{\prime} \cdot \alpha\left(1_{A[1]}\right) .
\end{aligned}
$$

Using the identity above, it follows that

$$
\begin{aligned}
m^{\prime} \cdot \beta\left(1_{A[0]}\right) a_{[0]}^{\prime} \otimes \gamma\left(c \cdot 1_{A[1]}\right) \cdot a_{[1]}^{\prime} & =\varepsilon(c) m_{[0]}^{\prime} \cdot \beta\left(1_{A[0]}\right) a_{[0]}^{\prime} \otimes m_{[1]}^{\prime} \cdot \alpha\left(1_{A[1]}\right) a_{[1]}^{\prime} \\
& \stackrel{(2.11)}{=} \varepsilon(c) m_{[0]}^{\prime} \cdot 1_{A^{\prime}[0]} a_{[0]}^{\prime} \otimes m_{[1]}^{\prime} \cdot 1_{A^{\prime}[1]} a_{[1]}^{\prime} \\
& \stackrel{(2.1)}{=} \varepsilon(c) m_{[0]}^{\prime} \cdot a_{[0]}^{\prime} \otimes m_{[1]}^{\prime} \cdot a_{[1]}^{\prime} \\
& =\rho_{M^{\prime}} \circ \delta_{M^{\prime}}\left(\left(m^{\prime} \cdot \beta\left(1_{A[0]}\right) \otimes c \cdot 1_{A[1]}\right) \otimes_{A} a^{\prime}\right) .
\end{aligned}
$$

This is what we need to show. We can check $\eta$ and $\delta$ defined above are all natural transformations and they satisfy

$$
G\left(\delta_{M^{\prime}}\right) \circ \eta_{G\left(M^{\prime}\right)}=I, \quad \delta_{F(M)} \circ F\left(\eta_{M}\right)=I,
$$

for all $M \in \mathcal{M}(H)_{A}^{C}$ and $M^{\prime} \in \mathcal{M}\left(H^{\prime}\right)_{A^{\prime}}^{C^{\prime}}$.

The proof of Theorem is completed.

REMARK 2.8. We consider $(H, A, k)$ and the map $l_{H}, l_{B}$ and $\varepsilon_{C}: C \rightarrow k$. Now $\mathcal{M}(H)_{A}\left(\right.$ or $\left.\mathcal{U}_{A}\right)$, the category of right $A$-modules, and $F$ is the functor which forgets the $C$-comodule structures. From Theorem 2.7, $G\left(M^{\prime}\right)=\left\{m^{\prime} \cdot 1_{A[0]} \otimes c \cdot 1_{A[1]}\right\}=\overline{M^{\prime} \otimes C}$ with structure maps

$$
\begin{gathered}
\rho_{G\left(M^{\prime}\right)}\left(m^{\prime} \cdot A[0]\right. \\
\left.\left(m^{\prime} \cdot 1_{A[0]} \otimes c \cdot 1_{A[1]}\right)=m^{\prime} \cdot 1_{A[1]}\right) \cdot a=m^{\prime} \cdot a_{[0][0]} \otimes c \cdot c_{[1]},
\end{gathered}
$$

for all $a \in A$ and $M^{\prime} \in \mathcal{M}(H)_{A}$. The unit $\eta$ of the adjunction $(F, G)$ is given by $\eta^{M^{\prime}}: M^{\prime} \rightarrow G\left(M^{\prime}\right)$

$$
\eta^{M^{\prime}}\left(m^{\prime}\right)=m_{[0]}^{\prime} \cdot 1_{A[0]} \otimes m_{[1]}^{\prime} \cdot 1_{A[1]} .
$$




\section{Integral of partial Doi-Hopf datum}

Definition 3.1. Let $(H, A, C)$ be a partial Doi-Hopf datum. A $k$-linear map

$$
\theta: C \otimes C \rightarrow A
$$

is called a normalized A-integral, if $\theta$ satisfies the following conditions:

$$
\begin{gathered}
c_{2} \cdot 1_{A[1] 3} \otimes 1_{A[0]} \theta\left(d \cdot 1_{A[1] 1} \otimes c_{1} \cdot 1_{A[1] 2}\right) \\
=c_{2} \cdot 1_{A[1]} \otimes 1_{A[0][0][0]} \theta\left(d \cdot 1_{A[0][0][1]} \otimes c_{1} \cdot 1_{A[0][1]}\right) \\
=d_{1} \cdot 1_{A[0][1]} \theta\left(d_{2} \cdot 1_{A[1]} \otimes c\right)_{A[1]} \otimes 1_{A[0][0]} \theta\left(d_{2} \cdot 1_{A[1]} \otimes c\right)_{[0]}, \\
\theta\left(c_{1} \otimes c_{2}\right)=1_{A} \varepsilon(c), \\
a_{[0][0]} \theta\left(c \cdot a_{[0][1]} \otimes d \cdot a_{[1]}\right)=\theta(c \otimes d) a,
\end{gathered}
$$

for all $a \in A$ and $c, d \in C$.

Theorem 3.2. For any partial Doi-Hopf datum $(H, A, C)$, the following assertions are equivalent,

(1) $\eta$ in Remark 2.8 is a split natural monomorphism.

(2) The forgetful functor $F$ is separable.

(3) There exists a normalized A-integral $\theta: C \otimes C \rightarrow A$.

Proof. $\quad(1) \Longleftrightarrow(2)$ follows by Rafael Theorem ([R]).

$(3) \Longrightarrow(1)$. For any partial Doi-Hopf module $M$, we define

$$
v^{M}: \overline{M \otimes C} \rightarrow M, v^{M}\left(m \cdot 1_{A[0]} \otimes c \cdot 1_{A[1]}\right)=m_{[0]} \theta\left(m_{[1]} \otimes c\right),
$$

for all $m \in M$ and $c \in C$.

Now, we shall check that $v^{M} \in \mathcal{M}(H)_{A}^{C}$. In fact, for all $m \in M, c \in C$ and $a \in A$,

$$
\begin{aligned}
v^{M}\left(\left(m \cdot 1_{A[0]} \otimes c \cdot 1_{A[1]}\right) \cdot a\right) & =v^{M}\left(m \cdot a_{[0]} \otimes c \cdot a_{[1]}\right) \\
& =\left(m \cdot a_{[0]}\right]_{[0]} \theta\left(\left(m \cdot a_{[0]}\right)_{[1]} \otimes c \cdot a_{[1]}\right) \\
& \stackrel{(2.8)}{=} m_{[0]} \cdot a_{[0][0]} \theta\left(m_{[1]} \cdot a_{[0][1]} \otimes c \cdot a_{[1]}\right) \\
& \stackrel{(3.3)}{=} m_{[0]} \theta\left(m_{[1]} \otimes c\right) a \\
& =v^{M}\left(m \cdot 1_{A[0]} \otimes c \cdot 1_{A[1]}\right) \cdot a .
\end{aligned}
$$

Hence it is a morphism of $A$-module. Next, we shall check that $v$ is a 
morphism of $C$-comodule. It is sufficient to check that

$$
\rho_{M} \circ v^{M}=\left(v^{M} \otimes \imath\right) \circ \rho_{G(M)}
$$

holds. For all $m \in M$ and $c \in C$, we have

$$
\begin{aligned}
& \rho_{M} \circ v^{M}\left(m \cdot 1_{A[0]} \otimes c \cdot 1_{A[1]}\right) \\
= & \rho_{M}\left(m_{[0]} \cdot \theta\left(m_{[1]} \otimes c\right)\right) \\
= & \left(m_{[0]} \cdot \theta\left(m_{[1]} \otimes c\right)\right)_{[0]} \otimes\left(m_{[0]} \cdot \theta\left(m_{[1]} \otimes c\right)\right)_{[1]} \\
\stackrel{(2.8)}{=} & m_{[0][0]} \cdot \theta\left(m_{[1]} \otimes c\right)_{[0]} \otimes m_{[0][1]} \cdot \theta\left(m_{[1]} \otimes c\right)_{[1]} \\
\stackrel{(2.7)}{=} & m_{[0]} \cdot 1_{[0][0]} \theta\left(m_{[1] 2} \cdot 1_{[1]} \otimes c\right)_{[0]} \otimes m_{[1] 1} \cdot 1_{[0][1]} \theta\left(m_{[1] 2} \cdot 1_{[1]} \otimes c\right)_{[1]}
\end{aligned}
$$

and

$$
\begin{aligned}
& \left(v^{M} \otimes \imath\right) \circ \rho_{G(M)}\left(m \cdot 1_{A[0]} \otimes c \cdot 1_{A[1]}\right) \\
= & \left(v^{M} \otimes \imath\right)\left(m \cdot 1_{A[0][0]} \otimes c_{1} \cdot 1_{A[0][1]} \otimes c_{2} \cdot 1_{A[1]}\right) \\
= & v^{M}\left(m \cdot 1_{A[0][0]} \otimes c_{1} \cdot 1_{A[0][1]}\right) \otimes c_{2} \cdot 1_{A[1]} \\
= & \left(m \cdot 1_{A[0][0]}\right)_{[0]} \theta\left(\left(m \cdot 1_{A[0][0]}\right)_{[1]} \otimes c_{1} \cdot 1_{A[0][1]}\right) \otimes c_{2} \cdot 1_{A[1]} \\
= & m_{[0]} \cdot 1_{A[0]}^{\prime} 1_{A[0][0]} \theta\left(m_{[1]} \cdot 1_{A[0][1]} \otimes c_{1} \cdot 1_{A[1]}\right) \otimes c_{2} \cdot 1_{A[1]}^{\prime} \\
= & m_{[0]} \cdot 1_{A[0]} \theta\left(m_{[1]} \otimes c_{1}\right) \otimes c_{2} \cdot 1_{A[1]} .
\end{aligned}
$$

Using (3.1), we can get the desired result. For all $m \in M$, Since

$$
\begin{aligned}
v^{M} \circ \eta_{M}(m) & =v^{M}\left(m_{[0]} \otimes m_{[1]}\right) \\
& =m_{[0][0]} \theta\left(m_{[0][1]} \otimes m_{[1]}\right) \\
& \stackrel{(2.7)}{=} m_{[0]} \cdot 1_{A[0][0]} \theta\left(m_{[1] 1} \cdot 1_{[0][1]} \otimes m_{[1] 2} \cdot 1_{A[1]}\right) \\
& =m_{[0]} \cdot \theta\left(m_{[1] 1} \otimes m_{[1] 2}\right) \\
& \stackrel{(3.2)}{=} m_{[0]} \varepsilon\left(m_{[1]}\right)=m .
\end{aligned}
$$

So it follows that $v$ splits $\eta$. It is evidently natural.

$(1) \Longrightarrow(3)$. We consider the following partial Doi-Hopf module $G(A)$. Evaluating at this object, the retraction $v$ of the unit $\eta$ yields a morphism

$$
v^{G(A)}: \overline{A \otimes C \otimes C} \rightarrow \overline{A \otimes C},
$$

where $\overline{A \otimes C \otimes C}=\left\langle a 1_{[0][0]} \otimes c \cdot 1_{A[0][1]} \otimes d 1_{A[1]}, a \in A, c, d \in C>\right.$. From $v \circ \eta=I$, we have

$$
v^{G(A)}\left(a 1_{A[0][0]} \otimes c_{1} \cdot 1_{A[0][1]} \otimes c_{2} \cdot 1_{A[1]}\right)=a \otimes c .
$$


It can be used to construct $\theta$ as follows:

$$
\begin{gathered}
\theta: C \otimes C \rightarrow A \\
\theta(c \otimes d)=\left(l \otimes \varepsilon_{C}\right) \circ v^{G(A)}\left(1_{A[0][0]} \otimes c \cdot 1_{A[0][1]} \otimes d \cdot 1_{A[1]}\right) .
\end{gathered}
$$

For all $c \in C$, since

$$
\begin{aligned}
\theta\left(c_{1} \otimes c_{2}\right) & =\left(l \otimes \varepsilon_{C}\right) \circ v^{G(A)}\left(1_{A[0][0]} \otimes c_{1} \cdot 1_{A[0][1]} \otimes c_{2} \cdot 1_{A[1]}\right) \\
& =\left(i d_{A} \otimes \varepsilon_{C}\right)\left(1_{A} \otimes c\right)=1_{A} \varepsilon_{C}(c) .
\end{aligned}
$$

Hence (3.2) holds. It can be seen to obey (3.3) by naturality and the $A$ module map property of $v$.

The verification of (3.1) is more involved. For any $C$-comodule $M$, we consider the partial Doi-Hopf module $M \otimes A$. The $A$-actions and $C$-coaction are defined as follows:

$$
\left\{\begin{array}{l}
(m \otimes a) \cdot b=m \otimes a b, \\
\rho^{M \otimes A}(m \otimes a)=m_{[0]} \otimes a_{[0]} \otimes m_{[1]} \cdot a_{[1]},
\end{array}\right.
$$

for all $m \in M, a, b \in A$. For $C$-comodule $C$, there is a partial Doi-Hopf module $C \otimes A$ and the map

$$
\xi: C \otimes A \rightarrow \overline{A \otimes C}, \xi(c \otimes a)=a_{[0]} \otimes c \cdot a_{[1]}
$$

induces a morphism of partial Doi-Hopf modules $C \otimes A \rightarrow \overline{A \otimes C}$. Thus by naturality of $v$, we have the following commutative diagram

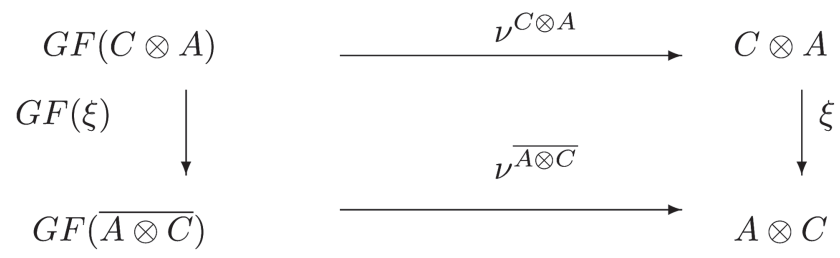

Explicitly, for all $a \in A, c, d \in C$, we have

(3.4) $\xi_{\circ} v^{C \otimes A}\left(c \otimes a 1_{A[0]} \otimes d \cdot 1_{A[1]}\right)=\left(v^{\overline{A \otimes C}}\right)\left(a_{[0]} 1_{A[0][0]} \otimes c \cdot a_{[1]} 1_{A[0][1]} \otimes d 1_{A[1]}\right)$.

We consider next the following partial Doi-Hopf module $C \otimes C$ with partial $C$-coaction given by comultiplication in the second factor. Then

$$
\chi=\Delta \otimes \imath: C \otimes A \rightarrow C \otimes C \otimes A, \chi(c \otimes a)=c_{1} \otimes c_{2} \otimes a
$$

induces a morphism of partial Doi-Hopf modules $C \otimes A \rightarrow C \otimes C \otimes A$. Thus 
by naturality of $v$, the following diagram

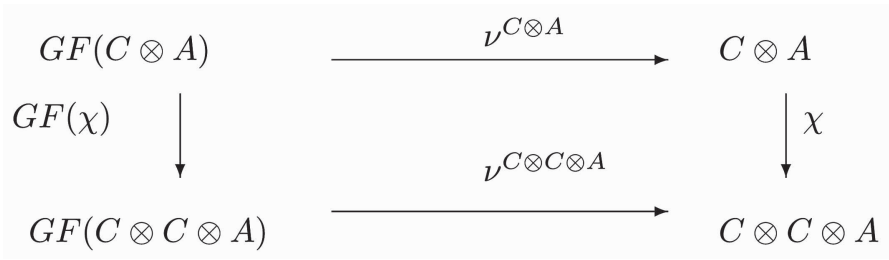

The commutative diagram above is equivalent to

$$
\chi \circ v^{C \otimes A}\left(c \otimes a 1_{A[0]} \otimes d \cdot 1_{A[1]}\right)=v^{C \otimes C \otimes A}\left(c_{1} \otimes c_{2} \otimes a 1_{A[0]} \otimes d \cdot 1_{A[1]}\right),
$$

for all $c, d \in C$ and $a \in A$. Finally, for any $c \in C$, the map

$$
f_{c}: C \otimes A \rightarrow C \otimes C \otimes A, \quad d \otimes a \mapsto c \otimes d \otimes a
$$

induces a morphism of partial Doi-Hopf modules $C \otimes A \rightarrow C \otimes C \otimes A$. Hence by naturality of $v$, we have

$$
c \otimes v^{C \otimes A}\left(e \otimes a 1_{A[0]} \otimes d \cdot 1_{A[1]}\right)=v^{C \otimes C \otimes A}\left(c \otimes e \otimes a 1_{A[0]} \otimes d \cdot 1_{A[1]}\right),
$$

for all $c, e, d \in C$ and $a \in A$. From (3.5) and (3.6),

$$
\chi \circ v^{C \otimes A}\left(c \otimes a 1_{A[0]} \otimes d \cdot 1_{A[1]}\right)=c_{1} \otimes v^{C \otimes A}\left(c_{2} \otimes a 1_{A[0]} \otimes d \cdot 1_{A[1]}\right),
$$

for all $c, d \in C$ and $a \in A$.

From $v^{G(A)}$ being $C$-colinear, it follows that

$$
\begin{aligned}
& \rho^{G(A)} \circ v^{G(A)}\left(1_{A[0][0]} \otimes c \cdot 1_{A[0][1]} \otimes d \cdot 1_{A[1]}\right) \\
& \quad=v^{G(A)}\left(1_{A[0][0][0]} \otimes c \cdot 1_{A[0][0][1]} \otimes d_{1} \cdot 1_{A[0][1]}\right) \otimes d_{2} \cdot 1_{A[1]},
\end{aligned}
$$

for all $c, d \in C$.

For all $c, d \in C$, since

$$
\begin{aligned}
& c_{2} \cdot 1_{A[1]} \otimes 1_{A[0][0] 00} \theta\left(d \cdot 1_{A[0][0][1]} \otimes c_{1} \cdot 1_{A[0][1]}\right) \\
= & c_{2} \cdot 1_{A[1] 3} \otimes 1_{A[0]} \theta\left(d \cdot 1_{A[1] 1} \otimes c_{1} \cdot 1_{A[1] 2}\right) \\
= & \tau_{A, C} \circ\left(\imath \otimes \varepsilon_{C} \otimes \imath\right)\left(v^{G(A)}\left(1_{A[0]} 1_{A[0][0]}^{\prime} \otimes d \cdot 1_{A[1] 1} 1_{[0][1]}^{\prime} \otimes c_{1} \cdot 1_{A[1] 2} 1_{A[1]}^{\prime}\right) \otimes c_{2} \cdot 1_{A[1] 3}\right) \\
= & \tau_{A, C} \circ\left(l \otimes \varepsilon_{C} \otimes \imath\right)\left(v^{G(A)}\left(1_{A[0][0][0]} \otimes d \cdot 1_{A[0][0][1]} \otimes c_{1} \cdot 1_{A[0][1]}\right) \otimes c_{2} \cdot 1_{A[1]}\right) \\
= & \tau_{A, C} \circ\left(\imath \otimes \varepsilon_{C} \otimes \imath\right) \rho_{G(A)} \circ v^{G(A)}\left(1_{A[0][0]} \otimes d \cdot 1_{A[0][1]} \otimes c \cdot 1_{A[1]}\right) \\
= & \tau_{A, C} \circ v^{G(A)}\left(1_{A[0][0]} \otimes d \cdot 1_{A[0][1]} \otimes c \cdot 1_{A[1]}\right),
\end{aligned}
$$

where the second equals is followed from $v$ being a left $A$-module. In fact, for 
all $a \in A$, the map

$$
f_{a}: \overline{A \otimes C} \rightarrow \overline{A \otimes C}, f_{a}\left(b 1_{A[0]} \otimes c \cdot 1_{A[1]}\right)=a b 1_{A[0]} \otimes c \cdot 1_{A[1]}
$$

is a morphism in the category $\mathcal{U}_{A}^{C}$. Hence by naturality of $v$, we have that $v$ is a left $A$-module. Since

$$
\begin{aligned}
& d_{1} \cdot 1_{A[0][1]} \theta\left(d_{2} \cdot 1_{A[1]} \otimes c\right)_{[1]} \otimes 1_{A[0][0]} \theta\left(d_{2} \cdot 1_{A[1]} \otimes c\right)_{[0]} \\
= & d_{1} \cdot\left(1_{A[0]} \theta\left(d_{2} \cdot 1_{A[1]} \otimes c\right)\right)_{[1]} \otimes\left(1_{A[0]} \theta\left(d_{2} \cdot 1_{A[1]} \otimes c\right)\right)_{[0]} \\
= & d_{1} \cdot\left(1_{A[0]}\left(l \otimes \varepsilon_{C}\right) \circ v^{G(A)}\left(1_{A[0][0]}^{\prime} \otimes d_{2} \cdot 1_{A[1]} 1_{A[0][1]}^{\prime} \otimes c \cdot 1_{A[1]}^{\prime}\right)\right)_{[1]} \\
& \otimes\left(1_{A[0]}\left(l \otimes \varepsilon_{C}\right) \circ v^{G(A)}\left(1_{A[0][0]}^{\prime} \otimes d_{2} \cdot 1_{A[1]} 1_{A[0][1]}^{\prime} \otimes c \cdot 1_{A[1]}^{\prime}\right)\right)_{[0]} \\
= & d_{1} \cdot\left(\left(l \otimes \varepsilon_{C}\right) \circ v^{G(A)}\left(1_{A[0]} 1_{A[0][0]}^{\prime} \otimes d_{2} \cdot 1_{A[1]} 1_{A[0][1]}^{\prime} \otimes c \cdot 1_{A[1]}^{\prime}\right)\right)_{[1]} \\
& \otimes\left(\left(l \otimes \varepsilon_{C}\right) \circ v^{G(A)}\left(1_{A[0]} 1_{A[0][0]}^{\prime} \otimes d_{2} \cdot 1_{A[1]} 1_{A[0][1]}^{\prime} \otimes c \cdot 1_{A[1]}^{\prime}\right)\right)_{[0]} \\
\stackrel{(3.4)}{=} & d_{1} \cdot\left(\left(l \otimes \varepsilon_{C}\right) \circ \xi \circ v^{C \otimes A}\left(d_{2} \otimes \cdot 1_{A[1]} 1_{A[0]} \otimes c \cdot 1_{A[1]}\right)\right)_{[1]} \\
& \otimes\left(\left(l \otimes \varepsilon_{C}\right) \circ \xi \circ v^{C \otimes A}\left(d_{2} \otimes \cdot 1_{A[0]} \otimes c \cdot 1_{A[1]}\right)\right)_{[0]}
\end{aligned}
$$

Let $v^{C \otimes A}\left(d \otimes a 1_{A[0]} \otimes c \cdot 1_{A[1]}\right)=c_{i} \otimes a_{i}$. Then

$$
\begin{gathered}
d_{1} \cdot\left(\left(l \otimes \varepsilon_{C}\right) \circ \xi \circ v^{C \otimes A}\left(d_{2} \otimes \cdot 1_{A[1]} 1_{A[0]} \otimes c \cdot 1_{A[1]}\right)\right)_{[1]} \\
\otimes\left(\left(l \otimes \varepsilon_{C}\right) \circ \xi \circ v^{C \otimes A}\left(d_{2} \otimes \cdot 1_{A[0]} \otimes c \cdot 1_{A[1]}\right)\right)_{[0]} \\
\stackrel{(3.7)}{=} c_{i 1} \cdot\left(\left(l \otimes \varepsilon_{C}\right) \circ \xi\left(c_{i 2} \otimes a_{i}\right)\right)_{[1]} \otimes\left(\left(l \otimes \varepsilon_{C}\right) \circ \xi\left(c_{i 2} \otimes a_{i}\right)\right)_{[0]} \\
=c_{i 1} \cdot a_{i[1]} \otimes a_{i[0]}=\sum \tau_{A, C} \circ \xi\left(c_{i} \otimes a_{i}\right) \\
=\sum \tau_{A, C} \circ \xi \circ v^{C \otimes A}\left(d \otimes a 1_{A[0]} \otimes c \cdot 1_{A[1]}\right) .
\end{gathered}
$$

Hence we can get (3.1) by using (3.4).

\section{Applications}

\section{1 - Maschke-type Theorems for partial Doi-Hopf modules}

Since separable functors reflect well the semisimplicity of the objects of a categogy, by Theroem 3.2, we will prove the Maschke-type theorems for partial Doi-Hopf modules.

Corollary 4.1. Let $(H, A, C)$ be a partial Doi-Hopf datum, and $M, N \in \mathcal{U}_{A}^{C}$. Suppose that there exists a total integral $\theta: C \otimes C \rightarrow A$. Then a monomorphism (resp. epimorphism) $f: M \rightarrow N$ splits in $\mathcal{U}_{A}^{C}$, if the monomorphism (resp. epimorphism) $f$ splits as an A-module morphism. 


\section{2 - Partial relative modules}

Let $H$ be a Hopf algebra and $A$ a partial right $H$-comodule algebra. Then the threetuple $(H, A, H)$ is a partial Doi-Hopf datum. The category $\mathcal{M}(H)_{A}^{H}$ is called a partial $(H, A)$-Hopf module category and denoted by $\mathcal{U}_{A}^{H}$.

Corollary 4.2. Let $H$ be a Hopf algebra and $A$ a partial right $H$ comodule algebra. Then the following statements are equivalent:

(1) The forgetful functor $F: \mathcal{U}_{A}^{H} \rightarrow \mathcal{U}_{A}$ is separable,

(2) There exists a normalized A-integral $\theta: H \otimes H \rightarrow A$.

We will now introduce the partial total integral for the partial right $H$ comodule algebra, and investigate the difference between the partial total integral and the total integral in sense of Doi.

Proposition 4.3. Let $H$ be a Hopf algebra and $A$ a partial right $H$-comodule algebra. If $\theta: H \otimes H \rightarrow k$ is a normalized $A$-integral for $(H, A, H)$, the $k$-linear map

$$
\varphi: H \rightarrow A, \varphi(h)=\theta\left(1_{H} \otimes h\right),
$$

for all $h \in H$, satisfies the relations:

$$
\begin{gathered}
\varphi(h)_{[0]} \otimes \varphi(h)_{[1]}=\varphi\left(h_{1}\right) 1_{A[0]} \otimes h_{2} 1_{A[1]}, \\
\varphi\left(1_{H}\right)=1_{A} .
\end{gathered}
$$

Proof. Notice first that $\varphi\left(1_{H}\right)=\theta\left(1_{H} \otimes 1_{H}\right)=\varepsilon_{H}\left(1_{H}\right) 1_{A}=1_{A}$. Since

$$
\begin{aligned}
& h_{2} 1_{A[1]} \otimes \theta\left(g \otimes h_{1}\right) 1_{A[0]} \\
& =h_{2} 1_{A[1]} \otimes 1_{A[0][0][0]} \theta\left(g 1_{A[0][0][1]} \otimes h_{1} 1_{A[0][1]}\right) \\
& =g_{1} 1_{A[0][1]} \theta\left(g_{2} 1_{A[1]} \otimes h\right)_{[1]} \otimes 1_{A[0][0]} \theta\left(g_{2} 1_{A[1]} \otimes h\right)_{[0]} \\
& =g_{1}\left(1_{A[0]} \theta\left(g_{2} 1_{A[1]} \otimes h\right)\right)_{[1]} \otimes\left(1_{A[0]} \theta\left(g_{2} 1_{A[1]} \otimes h\right)\right)_{[0]} \\
& =g_{1}\left(\theta\left(g_{2} \otimes h\right)\right)_{[1]} \otimes\left(\theta\left(g_{2} \otimes h\right)\right)_{[0]}
\end{aligned}
$$

It follows by taking $g=1_{H}$ that

$$
h_{2} 1_{A[1]} \otimes \theta\left(1_{H} \otimes h_{1}\right) 1_{A[0]}=\theta\left(1_{H} \otimes h\right)_{[1]} \otimes \theta\left(1_{H} \otimes h\right)_{[0]} .
$$

So (4.1) holds.

Definition 4.4. Let $H$ be a Hopf algebra and $A$ a right partial $H$-comodule algebra. A $k$-linear map $\varphi: H \rightarrow A$ is call a partial total integral for $(H, A)$, if $\varphi$ satisfies the conditions (4.1) and (4.2). 
REMARK 4.5. If $1_{A[0]} \otimes 1_{A[1]}=1_{A} \otimes 1_{H}$, then the right partial $H$-comodule algebra $A$ is just the ordinary right $H$-comodule algebra, and the partial total integral is the same with the total integral in sense of Doi in (D2).

Let $\varphi: H \rightarrow A$ be the total integral for the partial right $H$-coalgebra $A$, and define

$$
\theta: H \otimes H \rightarrow A, \theta(h \otimes g)=1_{A[0]} \varphi\left(g S^{-1}\left(1_{A[1]} h\right)\right),
$$

for all $g, h \in H$.

THeORem 4.6. Let $A$ be a partial right $H$-comodule algebra and $\varphi: H \rightarrow A$ be a partial total integral. If

$$
\begin{gathered}
g \varphi(h)_{[1]} \otimes \varphi(h)_{[0]}=\varphi(h)_{[1]} g \otimes \varphi(h)_{[0]}, \\
\varphi(h) \in Z(A) \text { (the center of } A), 1_{A[0]} \varphi\left(S^{-1}\left(1_{[1]}\right)\right)=1_{A},
\end{gathered}
$$

Then $\theta$ is a normalized A-integral.

Proof. For all $a \in A$ and $g, h \in H$, one has

$$
\begin{aligned}
a_{[0][0]} \theta\left(g a_{[0][1]} \otimes h a_{[1]}\right) & =a_{[0][0]} 1_{A[0]} \varphi\left(h a_{[1]} S^{-1}\left(1_{A[1]} g a_{[0][1]}\right)\right) \\
& =a_{[0]} 1_{A[0]} \varphi\left(h a_{[1] 2} S^{-1}\left(g a_{[1] 1} 1_{A[1]}\right)\right) \\
& =1_{A[0]} \varphi\left(h S^{-1}\left(g 1_{A[1]}\right)\right) a \\
& =\theta(g \otimes h)
\end{aligned}
$$

and

$$
\begin{aligned}
& g_{1}\left(\theta\left(g_{2} \otimes h\right)\right)_{[1]} \otimes\left(\theta\left(g_{2} \otimes h\right)\right)_{[0]} \\
& =g_{1} 1_{A[0] 1]]} \varphi\left(h S^{-1}\left(1_{A[1]} g_{2}\right)\right)_{[1]} \otimes 1_{A[0][0]} \varphi\left(h S^{-1}\left(1_{A[1]} g_{2}\right)\right)_{[0]} \\
& =\varphi\left(h S^{-1}\left(1_{A[1]} g_{2}\right)\right)_{[1]} g_{1} 1_{A[0][1]} \otimes 1_{A[0][0]} \varphi\left(h S^{-1}\left(1_{A[1]} g_{2}\right)\right)_{[0]} \\
& =h_{2} S^{-1}\left(1_{A[1]} g_{2}\right)_{2} 1_{A[1]}^{\prime} g_{1} 1_{A[0][1]} \otimes 1_{A[0][0]} \varphi\left(h_{1} S^{-1}\left(1_{A[1]} g_{2}\right)_{1}\right) 1_{A[0]}^{\prime} \\
& =h_{2} S^{-1}\left(1_{A[1] 1}\right) S^{-1}\left(g_{2}\right) g_{1} 1_{A[0][1]} \otimes 1_{A[0][0]} \varphi\left(h_{1} S^{-1}\left(1_{A[1] 2} g_{3}\right)\right) \\
& =h_{2} S^{-1}\left(1_{A[1] 2}\right) 1_{A[1] 1} 1_{A[1]}^{\prime} \otimes 1_{A[0]} 1_{A[0]}^{\prime} \varphi\left(h_{1} S^{-1}\left(1_{A[1] 3} g\right)\right) \\
& =h_{2} 1_{A[1]}^{\prime} \otimes 1_{A[0]} 1_{A[0]}^{\prime} \varphi\left(h_{1} S^{-1}\left(1_{A[1]} g\right)\right) \\
& =h_{2} 1_{A[1]} \otimes \theta\left(g \otimes h_{1}\right) 1_{A[0]} . \\
& \quad \theta\left(h_{1} \otimes h_{2}\right)=1_{A[0]} \varphi\left(h_{2} S^{-1}\left(1_{A[1]} h_{1}\right)\right) \\
& \quad=\varepsilon_{H}(h) 1_{A[0]} \varphi\left(S^{-1}\left(1_{A[1]}\right)\right)=\varepsilon_{H}(h) 1_{A}
\end{aligned}
$$

So $\theta$ is a normalized $A$-integral. 


\section{3 - Partial Doi-Hopf Datum $(H, k, H)$}

COROLlary 4.7. Under the assumptions of Example 2.5. Then the following statements are equivalent:

(1) The forgetful functor $F: \mathcal{M}(H)^{H} \rightarrow \mathcal{M}_{k}$ (the category of all vector spaces) is separable.

(2) $k$-linear map $\theta: H \otimes H \rightarrow k$ such that the following conditions are satisfied:

$$
\begin{gathered}
\theta\left(h_{1} \otimes h_{2}\right)=\varepsilon_{H}(h), \\
\theta(e h \otimes e g)=\theta(h \otimes g), \\
e h_{2} \theta\left(g \otimes h_{1}\right)=e g_{1} \theta\left(e g_{2} \otimes h\right) .
\end{gathered}
$$

Take $e=1$. Then the partial Doi-Hopf datum $(H, k, H)$ is just the DoiHopf datum, the category $\mathcal{M}(H)^{H}$ is the category $\mathcal{M}^{H}$ of $H$-Hopf module. Suppose that $\varphi$ is the right integral of $H^{*}$, the map $\theta: H \otimes H \rightarrow k$ is defined by

$$
\theta(h \otimes g)=\varphi\left(g S^{-1}(h)\right) .
$$

By the properties of the right integral $\varphi$, we can check that $\theta$ satisfies (4.3)(4.4).

Corollary 4.8. Let $H$ be a finite dimensional cosemisimple Hopf algebra. The forgetful functor $F: \mathcal{M}^{H} \rightarrow \mathcal{M}_{k}$ is separable.

Proof. Since $H$ is a finite dimensional cosemisimple Hopf algebra, it follows that there exists a right integral $\varphi \in H^{*}$ such that $\varphi\left(1_{H}\right)=1$. The desired total integral $\theta$ can be constructed by using $\varphi$.

Acknowledgment. The authors would like to thank the referee for the valuable suggestions and comments.

\section{REFERENCES}

[CJ] S. Caenepeel - K. Janssen, Partial (Co)Actions of hopf algebras and Partial Hopf-Galois Theory, Comm. Algebra, 36 (2008), pp. 2923-2946.

[CMI] S. CAenePEel - G. Militaru - B. Ion, Separable functors for the category of Doi-Hopf modules, Applications, Adv. Mathematics, 145 (1999), pp. 239-290.

[CR] S. CAenePeel - S. Raianu, Induction functors for the Doi-Koppinen unified Hopf modules, in Abelian Groups and Modules, pp. 73-94, Kluwer Acad. Publ., Dordrecht, 1995. 
[D1] Y. DoI, Unifying Hopf modules, J. Algebra, 153 (2) (1992), pp. 373-385.

[D2] Y. DoI, Algebras with total integrals, Comm. Algebra, 13 (1986), pp. 21372159.

[DE] M. Dokuchaev - R. Exel, Associativity of Crossed Products by Partial Actions, Enveloping Actions and Partial Representations, Trans. Amer. Math. Soc., 357 (2005), pp. 1931-1952.

[DEP] M. Dokuchaev - R. Exel - P. Piccione, Partial Representations and Partial Group Algebras, J. Algebra, 226 (2000), pp. 251-268.

[DFP] M. Dokuchaev - M. Ferrero - A. Pacques, Partial Galois theory of commutative rings, J. Pure Appl. Algebra, 208 (2007), pp. 77-87.

[DZ] M. Dokuchaev - N. Zhukavets, On Finite Degree Partial Partial Representations of Groups, J. Algebra, 274 (2000), pp. 309-334.

[E1] R. Exel, Twisted Partial Actions: A Classfication of Regular C*-Algebraic Bundles, Proc. London. Math. Soc., 74 (1997), pp. 417-443.

[E2] R. Exel, Circle Actions on $C^{*}$-Algebras, Partial Automorphisms and Generalized Pimsner-Voiculescu Exact Sequences, J. Funct. Anal., 122 (1994), pp. 361-401.

[R] M. D. RAFAEL, Separable functors revised, Comm. Algebra, 18 (1990), pp. 1445-1459.

[RT] D. E. RADFORD - J. TowBER, Yetter-Drinfeld categories associated to an arbitrary bialgebra, J. Pure Appl. Algebra, 87 (3) (1993), pp. 259-279.

[S] M. E. Sweedler, Hopf Algebras, Benjamin, New York, 1969.

[Y] D. N. YETTER, Quantum groups and representations of monoidal categories, Math. Proc. Cambridge Philos. Soc., 108 (2) (1990), pp. 261-290.

Manoscritto pervenuto in redazione il 13 Dicembre 2011. 